\title{
Nuevos registros y estado actual de la distribución de Imantodes inornatus Boulenger, 1896 (Serpentes: Dipsadidae) en Colombia
}

\section{New records and current status of the distribution of Imantodes inornatus Boulenger, 1896 (Serpentes: Dipsadidae) in Colombia}

\author{
Echavarría-R, Jonard ${ }^{1 *}$ Biol; Meneses-P, Elson² Biol; Jiménez-O, Alex ${ }^{3}$ Ph.D; \\ Palacios-M, Leison ${ }^{3}$ M.Sc; Rengifo-M, Jhon ${ }^{1}$ Ph.D. \\ ${ }^{1}$ Universidad Tecnológica del Chocó, Facultad de Ciencias Básicas y Exactas, Programa de Biología, \\ Grupo de Investigación en Herpetología, Colombia. \\ ${ }^{2}$ Universidad Industrial de Santander, Bucaramanga, Santander, Colombia. \\ ${ }^{3}$ Universidad Tecnológica del Choco, Facultad de Ciencias Básicas y Exactas, Programa de Biología, \\ Grupo de Investigación en Manejo y Gestión de la vida Silvestre del Chocó, Colombia.
}

\section{KEYWORDS:}

Biodiversity, conservation, Pacific region, taxonomy, Chocó, Colombia.

\section{ABSTRACT}

In order to document the current state of knowledge and geographical distribution of Imantodes inornatus, a review of specimens deposited in the Scientific Collection of Zoological Reference of Chocó-Herpetología (UTCH: COLZOOCH-H) and the Herpetological Collection of the Industrial University of Santander (UIS-R), as well as a meticulous search of records of the species published to date. We present here three new locations and an update of the geographic and altitudinal distribution of the species in Colombia, while two new habitats are now occupied by I. inornatus and some aspects of its conservation status are commented.

\section{PALABRAS CLAVE:}

Biodiversidad, conservación, región pacifica, taxonomía, Chocó, Colombia.

\section{RESUMEN}

Con el fin de documentar el estado de conocimiento y distribución geográfica actual de Imantodes inornatus, se llevó a cabo una revisión de ejemplares depositados en la Colección Científica de Referencia Zoológica del Chocó-Herpetología (UTCH: COLZOOCH-H) y la Colección Herpetológica de la Universidad Industrial de Santander (UIS-R), así como una búsqueda minuciosa de registros de la especie publicados a la fecha. Presentamos aquí tres nuevas localidades y una actualización de la distribución geográfica y altitudinal de la especie en Colombia, al tiempo que, se adicionan dos nuevos hábitats ocupados ahora por I. inornatus y se comentan algunos aspectos sobre su estado de conservación. 


\section{INTRODUCCIÓN}

Imantodes DUMERIL 1853, pertenece a la subfamilia Dipsadinae (PYRON et al. 2013 y ZHENG y WIENS, 2016) (UETZ y HOŠEK, 2017) y se caracteriza por tener cuerpos verticalmente comprimidos, cabeza notablemente visible y diferenciable del cuerpo, ojos grandes con pupilas elípticas, cuello desproporcionadamente largo y delgado (SAVAGE, 2002). En general sus especies tienen una vida secretiva debido a que son preferentemente nocturnas y arborícolas, con reproducción ovípara y se alimentan de anfibios y pequeños lagartos (SAVAGE, 2002; BOLAÑOS, 2009; NATERA-MUMAW et al., 2015). Se reconocen actualmente ocho especies en la región Neotropical (UETZ y HOŠEK, 2017), I. cenchoa (LINNAEUS, 1758), I. gemmistratus (COPE, 1861), I. tenuissimus (COPE, 1867), I. phantasma (MYERS, 1982), I. chocoensis (TORRES-CARVAJAL et al. 2012), I. guane (MISSASSI y PRUDENTE, 2015), I. lentiferus (COPE, 1894) e I. inornatus (BOULENGER, 1896). Aunque entre esas especies, sólo I. cenchoa e I. lentiferus son de amplia distribución (TORRESCARVAJAL et al. 2012; MISSASI y PRUDENTE, 2015), las cuales están distribuidas desde México (América Central) hasta Argentina (América del Sur) (MYERS, 1982; TORRES-CARVAJAL et al. 2012) a elevaciones desde el nivel del mar hasta 2.200 msnm (MYERS, 1982). Entre las ocho especies, seis están en Colombia (Cordillera Occidental y Oriental de los Andes, en diferentes ambientes (MYERS 1982; PÉREZ-SANTOS y MORENO, 1988; TORRES-CARVAJAL et al., 2012, JARAMILLO-MARTÍNEZ et al., 2013; MISSASI y PRUDENTE, 2015).

Imantodes inornatus se distribuye desde las tierras bajas en la vertiente del Caribe en el noroeste de Honduras hacia el Este de Panamá; y en la vertiente del Pacífico al suroeste de Costa Rica y sudoeste de Panamá; y desde el extremo este de Panamá hacia Ecuador. Habita en las zonas tropical y subtropical occidental en un rango altitudinal de 5-1.450 msnm (SAVAGE, 2002). En Colombia se puede encontrar en las tierras bajas de la región Pacífica, el norte de la cordillera Central y el valle del río Magdalena entre los 0 y 1.450 msnm (SUÁREZ y ALZATE-BASTO, 2014; DÍAZ-AYALA et al., 2016). En los departamentos Antioquia, Bolívar, Boyacá, Caldas y Valle del Cauca.

El presente trabajo da a conocer los primeros registros documentados para los departamentos del Chocó y Santander de I. Inornatus, a partir de una revisión de especímenes depositados en la Colección Científica de Referencia Zoológica del Chocó-Herpetología de la Universidad Tecnológica del Chocó (COLZOOCH-H) y la Colección Herpetológica de la Universidad Industrial de Santander (UIS-R) del género Imantodes, con base en seis ejemplares, tres en cada departamento. Presentamos aquí, tres nuevas localidades y una actualización de la distribución geográfica de esta especie en Colombia, ampliando su distribución geográfica hacia la vertiente pacífica de Colombia y Valle del Magdalena medio para los departamentos del Chocó y Santander, respectivamente.

\section{MATERIALES Y MÉTODOS}

Se revisaron 51 especímenes del género Imantodes depositados la Colección Científica de Referencia Zoológica del Chocó-Herpetología de la Universidad Tecnológica del Chocó (UTCH: COLZOOCH: N=48) y la Colección Herpetológica de la Universidad Industrial de Santander (MHN-UIS: N=3) (Anexo 1), cuya clasificación taxonómica había estado en duda durante varios años. La identificación de la ejemplares fue realizada siguiendo las descripciones de la especie propuestas en TORRES-CARVAJAL et al. (2012), MISSASSI y PRUDENTE (2015), ECHAVARRÍA-RENTERÍA et al. (2016); dicho material colectado se encuentra en buen estado de conservación. Adicionalmente a estos registros, se revisó la base de datos del Global Biodiversity Information Facility "GBIF" (http://data.gbif. org), de donde se obtuvieron registros de presencia de la especie para el territorio colombiano, registros publicados de Imantodes inornatus en literatura, y junto a nuestros registros fueron utilizados para construir un mapa de distribución actual de la especie en Colombia.

\section{RESULTADOS Y DISCUSIÓN}

Producto de la revisión del material se determinaron tres especies: I. cenchoa, I. chocoensis, e I. inornatus, siendo esta última objeto de esta investigación (Fig. 1 ), basados en seis especímenes identificados con número de colección UTCH: COLZOOCH-H: 0219, UTCH: COLZOOCH-H: 1499 y UTCH: COLZOOCH-H: 2222, en la Colección Herpetológica del Chocó, MHNUIS-R-3273, MHN-UIS-R-3277 y MHN-UIS-R-3278 en la colección Herpetológica de la Universidad Industrial de Santander, que habían sido asignados como Imantodes sp. Así mismo, dos ejemplares asociados a este mismo género en la Colección Herpetológica del Chocó presentaron dificultades para la determinación taxonómica, que probablemente podrían referirse a (Imantodes aff. lentiferus).

Imantodes inornatus fue inicialmente reportada para el Chocó por CASTAÑO et al. (2004), con base en un ejemplar (ICN 120), sin embargo, MISSASSI y PRUDENTE (2015) y MISSASSI et al. (2015) corroboraron su identificación y la asignan a $l$. chocoensis (ECHAVARRÍA-RENTERÍA et al., 2016). De acuerdo con la información obtenida, estos reportes representan los primeros registros formales 
de la especie para el departamento del Chocó. De igual forma, constituyen nuevos registros para el departamento de Santander-Colombia, sustentados por material museológico formales de la especie.

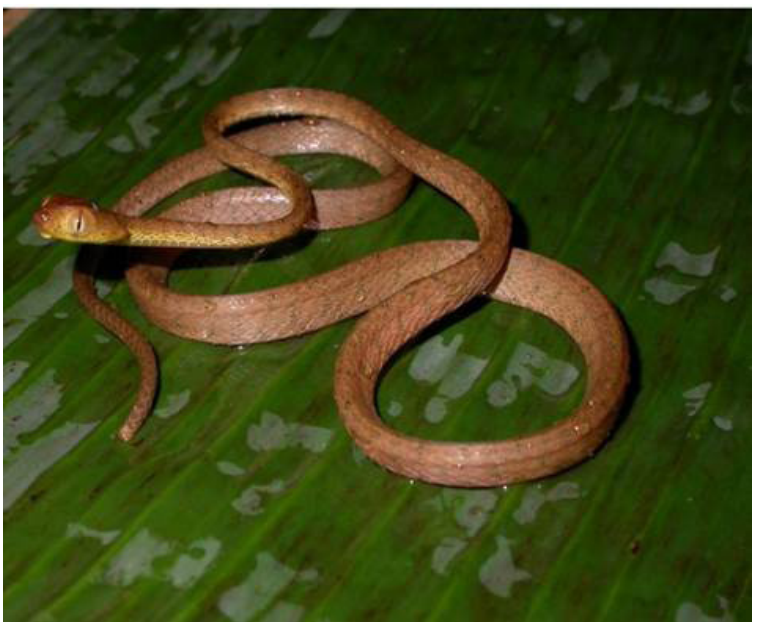

Figura 1. Espécimen en vida de I. inornatus, colectado en el corregimiento de Pacurita, municipio de Quibdó. ChocóColombia (5.683, -76.66, 53 m). Foto: Alex Mauricio JiménezOrtega.

El ejemplar de colección UTCH: COLZOOCH-H:0219, corresponde a un macho adulto, procedente del corregimiento de Pacurita, municipio de Quibdó (5.683,76.66). El Segundo ejemplar UTCH: COLZOOCH-H: 1499 , hace referencia a un macho juvenil, colectado el 25 de junio 2005 y tercer ejemplar UTCH: COLZOOCH-H:
2222, corresponde a una hembra adulta, colectada el 30 junio de 2014, estas dos últimas registradas en la localidad de Salero, municipio de Unión Panamericana (5.360, -76.64). Un cuarto ejemplar corresponde a tres individuos colectados el 10 de noviembre de 2014 en la vereda Sogamoso, municipio de Betulia, departamento de Santander (MHN-UIS-R-3273, MHNUIS-R-3277 y MHN-UIS-R-3278). Los nuevos registros aquí presentados amplían la distribución conocida de I. inornatus, que ahora se extiende desde Honduras hasta la provincia biogeográfica del Chocó, la cual hace parte del Hotspot o ecorregiones prioritarias una de las zonas más importantes en términos de conservación de la biodiversidad (Tumbes-Chocó-Magdalena) y la vertiente occidental de la cordillera Oriental en Colombia (Figura 1), con un distribución altitudinal entre 0 metros de elevación (DÍAZ-AYALA et al. 2016) y los 2.575 (Tabla. 1; Figura 2).

Las localidades de colecta en el departamento del Chocó corresponden a una zona de vida de Bosques Pluvial Tropical (bp-T), ubicadas en la zona centro del Chocó Biogeográfico-colombiano, caracterizadas por presentar los mayores volúmenes de precipitación promedio anual $8.000-14.000 \mathrm{~mm}$, temperatura que oscila entre $26^{\circ} \mathrm{C}$ a $28^{\circ} \mathrm{C}$ y una húmeda relativa de $95 \%$ (HOLDRIDGE, 1996, RANGEL-CH., 2004, BOLAÑOS et al., 2005). Las localidades del departamento de Santander se encuentran dentro de la formación de Bosque Seco Tropical (950 y 1.047 mm de precipitación anual) y corresponde a un régimen de distribución bimodal tetraestacional con bajas precipitaciones

Tabla 1. Lista completa de localidades de Imantodes inornatus en Colombia, basadas en datos de literatura y museos.

\begin{tabular}{|c|c|c|c|c|c|}
\hline Espécimen & Departamento & Municipio/Localidad & Coordenadas & Elevación (M) & Referencias \\
\hline MHUA-R 14873 & \multirow[t]{2}{*}{ Bolívar } & Norosi & $8.415,-74.22$ & 200 & \multirow{4}{*}{$\begin{array}{c}\text { Colección de Reptiles-Museo de } \\
\text { Herpetología de la Universidad de } \\
\text { Antioquía (MHUA-R) }\end{array}$} \\
\hline MHUA-R 14540 & & Anorí & $6.967,-75.05$ & 1535 & \\
\hline MHUA-R 14720 & \multirow[t]{2}{*}{ Antioquía } & Guadalupe & $6.836,-75.25$ & 1875 & \\
\hline- & & - & $6.176,-74.84$ & 898 & \\
\hline MLS 1142 & \multirow{4}{*}{ Chocó } & Quibdó /Tutunendo & $5.833,-76.66$ & 42 & $\begin{array}{c}\text { Colección de ofidios Museos de la } \\
\text { Salle Bogotá (MLS) }\end{array}$ \\
\hline $\begin{array}{l}\text { UTCH: } \\
\text { COLZOOCH-H } 0219\end{array}$ & & Quibdó/Pacurita & $5.683,-76.66$ & 53 & \multirow{3}{*}{$\begin{array}{l}\text { Colección Científica de Referencia } \\
\text { Zoológica del Chocó-Herpetología } \\
\text { (UTCH: COLZOOCH-H), } \\
\text { (ECHAVARRÍA-RENTERÍA, et al. } \\
\text { 2016) }\end{array}$} \\
\hline $\begin{array}{l}\text { UTCH: } \\
\text { COLZOOCH-H } 1499\end{array}$ & & \multirow{2}{*}{$\begin{array}{l}\text { Unión Panamericana/ } \\
\text { Salero }\end{array}$} & $5.360,-76.64$ & & \\
\hline $\begin{array}{l}\text { UTCH: } \\
\text { COLZOOCH-H } 2222\end{array}$ & & & $5.319-76.61$ & 30 & \\
\hline UIS-R-3273 & \multirow{3}{*}{ Santander } & & & & \multirow{3}{*}{$\begin{array}{l}\text { Colección Herpetológica de } \\
\text { la Universidad Industrial de } \\
\text { Santander (UIS-R) }\end{array}$} \\
\hline UIS-R-3277 & & $\begin{array}{l}\text { Betulia/Vereda } \\
\text { Sogamoso }\end{array}$ & $7.059,-73.38$ & 2575 & \\
\hline UIS-R-3278 & & & & 2515 & \\
\hline FUNTRO: 3046 & Valle del Cauca & Calima/Río Calima & $3.931,-76.67$ & 635 & $\begin{array}{c}\text { Funtropico-RFPR-Riobravo- } \\
\text { Paraguero }\end{array}$ \\
\hline \multirow[t]{2}{*}{ - } & \multirow[t]{2}{*}{ Boyacá } & Puerto Romeo & $5,833,-74.34$ & 661 & (DÍAZ-AYALA et al. 2016) \\
\hline & & Samaná & $5.406,-74.99$ & 1460 & \multirow{3}{*}{$\begin{array}{l}\text { Museo de Historia Natural de la } \\
\text { Universidad de Caldas (MHN-UC) }\end{array}$} \\
\hline \multirow[t]{2}{*}{ MHN-UC } & \multirow[t]{2}{*}{ Caldas } & Norcasia & $5.561,-74.88$ & 700 & \\
\hline & & Victoria & $5.302,-74.91$ & 750 & \\
\hline
\end{tabular}




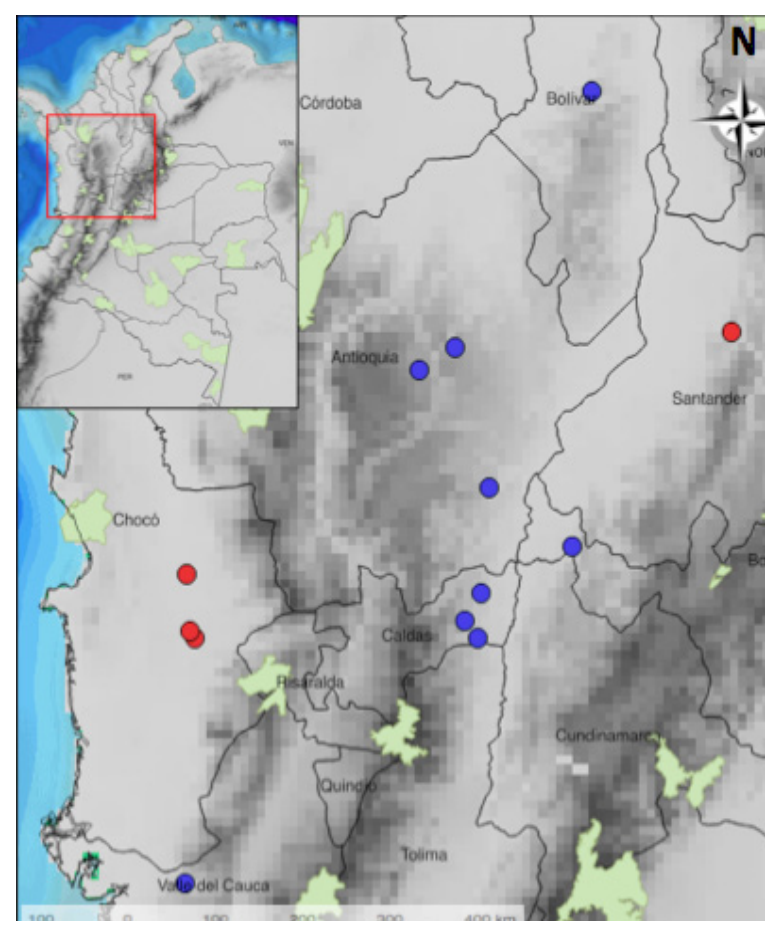

Figure 2. Distribución actual de Imantodes inornatus en Colombia, mostrando los antecedentes conocidos (círculos azules) y nuevos registros (círculos rojos) reportados aquí. Datum WGS84.

durante diciembre-febrero y junio-agosto y altas precipitaciones durante marzo-mayo y septiembrenoviembre (PINZÓN, 2014). Temperatura media anual de $24^{\circ} \mathrm{C}$ (FUNDACIÓN NATURA, 2010).

Referente al estado de conservación de I. inornatus, según VARGAS-ÁLVAREZ et al. (2016) se encuentra bajo la categoría de preocupación menor (LC), debido a los vacíos de información que existen sobre aspectos de su ecología, biología, taxonomía de las especies del género y sus relaciones filogenéticas. De igual forma, no se encuentran en ningún Apéndice CITES (2014).

El hallazgo de esta especie en el departamento del Chocó, constituye un hecho de suma importancia al incrementar el número de serpientes para esta porción del territorio colombiano, y se amplía la distribución geográfica de esta especies hacia el occidente y el norte de los Andes, lo cual refleja la importancia de conservar los bosques de la selva natural chocoana para preservar la mega diversa de fauna de serpientes de Colombia. Así mismo, la presencia de esta especie en el Valle del Magdalena medio en Santander, corrobora aún más, la dinámica o conexión entre la fauna de la llamada provincia Chocó-Magdalena, siendo esto un resultado importante en términos biogeográficos como los ya registrados previamente para la especie de serpiente Sibon annulatus (MENESES-PELAYO et al. 2016) reportado en estos dos departamentos de Colombia, la cual presenta similitudes respecto a su rango de distribución.

\section{Agradecimientos.}

A las comunidades de Pacurita (Quibdó), Salero (Unión Panamericana) y a la Vereda Sogamoso que permitieron realizar estas investigaciones. A las instituciones: Universidad Tecnológica del Chocó "Diego Luis Córdoba", y Universidad Industrial de Santander, Bucaramanga, por ser una vez más instituciones que apoyan las investigaciones, a todos los investigadores que aportaron con sus estudios. Y por último a el Grupo de Investigación en Herpetología de la Universidad Tecnológica del Chocó.

\section{Anexo 1.}

\section{Espécimenes examinados:}

$(n=6)$.

Pacurita-Cabí (5. 66583, -76.60577), 1 ঐ (UTCH:COLZOOCH-H: 0219). Unión Panamericana-Salero (5.360277778, -76.6458333; 5.319722222, -76.6177778), 2 우 (UTCH:COLZOOCH-H: 1499; UTCH:COLZOOCH-H: 2222).

Vereda Sogamoso (7.059, -73.38), 3 o (UIS-R-3273; UIS-R-3277; UIS-R-3278). (n=4). Pacurita-Cabí, Lloró (5. 67539, -76. 62086; 5.527222222, -76.55583333), 2 § (UTCH:COLZOOCH-H:0275; UTCH:COLZOOCH-H: 2933). Samurindó, Lloró (5.5875, -76.6541667; 5.527222222, -76.55583333), 2 ᄋ (UTCH:COLZOOCH-H:0318; UTCH:COLZOOCH-H: 2923).

$(n=39)$.

Cerro Tacarcuna, Nauca, Pacurita (Cabí), Salero, Samurindó, Lloró (5. 64952, -76. 57938; 5.60512, -77.01697; $5.686388889,-76.6333333 ; 5.32944444,-76.6275 ; 5.5925,-76.65 ; 5.527222222,-76.55583333), 12 \pi$ (UTCH:COLZOOCH-H: 0295; UTCH:COLZOOCH-H: 0583; UTCH:COLZOOCH-H: 1169; UTCH:COLZOOCH-H: 1559; UTCH:COLZOOCH-H: 1560; UTCH:COLZOOCH-H: 1648; UTCH:COLZOOCH-H: 1649; UTCH:COLZOOCH-H: 1650; UTCH:COLZOOCH-H: 1651; UTCH:COLZOOCH-H: 2637; UTCH:COLZOOCH-H: 2706; UTCH:COLZOOCH-H: 2839; UTCH:COLZOOCH-H: 2932. 
San Isidro, San José de Purré, San Martín de Purré, Samurindó, Salero, Pacurita (Cabí ), Unguía, San Roque, Angostura, Cerro Macarane (5.62639, -76.74884; 5.633333333, -76.55; 5. 64952, -76. 57938; 5.5875, -76.6541667, 5.980089, -76.88903; 5.360277778, -76.6458333; 5.686388889, -76.6333333; 5.704722222, -76.44027778; $5.319722222,-76.6177778 ; 8.025,7.715694444 ; 5.527222222,-76.55583333 ; 5.32944444,-76.6275$; $5.69583333,-76.6544444 ; 5.69583333,-76.6544444 ; 6.23888889,-77.3388889 ; 5.32944444,-76.6275) .27$ 우 (UTCH:COLZOOCH-H: 0078; UTCH:COLZOOCH-H: 0263; UTCH:COLZOOCH-H: 0293; UTCH:COLZOOCH-H: 0294; UTCH:COLZOOCH-H: 0317; UTCH:COLZOOCH-H: 1168; UTCH:COLZOOCH-H: 1181; UTCH:COLZOOCH-H: 1416; UTCH:COLZOOCH-H: 1420; UTCH:COLZOOCH-H: 1423; UTCH:COLZOOCH-H: 1431, UTCH:COLZOOCH-H: 1445; UTCH:COLZOOCH-H: 1478; UTCH:COLZOOCH-H: 1646; UTCH:COLZOOCH-H: 1647; UTCH:COLZOOCH-H: 2098; UTCH:COLZOOCH-H: 2128; UTCH:COLZOOCH-H: 2217; UTCH:COLZOOCH-H: 2245; UTCH:COLZOOCH-H: 2246; UTCH:COLZOOCH-H: 2448; UTCH:COLZOOCH-H: 2537; UTCH:COLZOOCH-H: 2636; UTCH:COLZOOCH-H: 2651; UTCH:COLZOOCH-H: 3021; UTCH:COLZOOCH-H: 3037).

\section{REFERENCIAS}

BOLAÑOS-ACOSTA, N.E. 2009. Imantodes cenchoa. En: Catálogo de biodiversidad de Colombia. Disponible en: http://www.siac.net.co/sib/catalogoespecies/especie.do?id. Consultado: 09-09-2017.

BOLAÑOS, R.; WATSON, V.; TOSI, J. 2005. Mapa ecológico de Costa Rica (Zonas de Vida), según el sistema de clasificación de zonas de vida del mundo de L.R. Holdridge), Escala 1:750 000. Centro Científico Tropical, San José, Costa Rica.

BOULENGER, G.A. 1896. Catalogue of the snakes in the British Museum, Vol. 3. London; Taylor y Francis.

CASTAÑO-MORA, O.V.; CÁRDENAS, G.; HERNÁNDEZ, E.; CASTRO, F. 2004. Reptiles en el Chocó biogeográfico de Colombia. En: Rangel-Ch, J O. (ed.). 2004. Colombia Diversidad Biótica, IV, El Chocó Biogeográfico/Costa Pacífica. Instituto de Ciencias Naturales de la Universidad Nacional de Colombia, Conservación Internacional, Bogotá.

CONVENCIÓN SOBRE COMERCIO INTERNACIONAL DE ESPECIES SILVESTRES DE FAUNA Y FLORA (CITES). Lista de Especies CITES. . Consultado: 09-09-2017. Disponible en: www.cites.org/esp/app/2014/SAppendices-2014-06-12.pdf

COPE, E.D. 1861. Contributions to the ophiology of Lower California, Mexico and Central America. Proceedings of the Academy of Natural Sciences of Philadelphia 13:292-306.

COPE, E.D. 1867. Quinta contribución a la herpetología de América tropical. Proceedings of the Academy of Natural Sciences of Philadelphia 18 (1866): 317-323.

COPE, E.D. 1894. On the species of Himantodes Dumeril and Bibron. American Naturalist 28: 612-614.

DÍAZ-AYALA, R.F.; ROJAS-MORALES, J.A.; ZULUAGA-ISAZA, J.C.; RAMÍREZ-CASTAÑO, V.A. 2016. Extensión de distribución y primeros registros para el departamento de caldas de la serpiente bejuquillo, Imantodes inornatus (Serpentes: Dipsadidae). En. Memoria del Primer Congreso de Herpetología. Medellín-Antioquia: 198.

DUMÉRIL, A.M.C. 1853 . Prodrome de la classification des reptiles Ophidiens. Mémoires de l'académie des Sciences, Paris 23:399-536.

ECHAVARRÍA-RENTERÍA, J.D.; RENTERÍA-M. L.E.; RENGIFO-MOSQUERA, J.T. 2016. Nuevo registro de Imantodes chocoensis (Squamata: Dipsadidae) en el Chocó Biogeográfico Colombiano. Revista Colombiana Ciencia Animal $8(1): 14-19$.

FUNDACIÓN NATURA. 2010. Propuesta Piloto de Restauración, Plan de Restauración Ecológica del Bosque Seco. Proyecto Hidroeléctrico El Quimbo. Bogotá D.C. Disponible en: http://www.natura.org.co/. Consultado: 09-09-2017.

HOLDRIDGE, L. 1996. Ecología basada en zonas de vida. San José: Instituto Interamericano de Cooperación para la Agricultura (IICA). Costa Rica. 
JARAMILLO-MARTÍNEZ, A.F.; VALENCIA-ZULETA, A.; CASTRO-HERRERA, F. 2013. Imantodes chocoensis Torres-Carvajal, Yánez-Muñoz, Quirola, Smith, and Almendáriz, 2012 (Squamata: Dipsadidae): First records from Colombia. Check List 9 (5):1070-1071.

LINNAEUS, C. 1758. Systema naturæ por regna tria naturæ, clases secundum, ordines, géneros, especies, cum characteribus, differentiis, synonymis, locis. Tomus I. Editio decima, reformata. Laurentii Salvii, Holmiæ. $10^{a}$ Edición.

MENESES-PELAYO, E.; ECHAVARRÍA-RENTERÍA, JD.; BAYONA-SERRANO1, J.D.; CAICEDO-PORTILLA, J.R.; RENGIFO-MOSQUERA, J. T. 2016. New records and an update of the distribution of Sibon annulatus (Colubridae: Dipsadinae: Dipsadini) for Colombia. Check List 12 (4):1931.

MISSASSI, A.F.R.; COSTA, C.L.; PRUDENTE, A.L.C. 2015. Range extension of the Chocoan blunt-headed vine snake: Imantodes chocoensis (Serpentes: Dipsadidae) in Northwestern Colombia. Salamandra 51 (3):269-272.

MISSASSI, A.F.R.; PRUDENTE, A.L.C. 2015. A new species of Imantodes Duméril, 1853 (Serpentes, Dipsadidae) from the Eastern Cordillera of Colombia Zootaxa 3980 (4):562-574.

MYERS, C.W. 1982. Blunt-headed vine snakes (Imantodes) in Panama, including a new species and other revisionary notes. American museum novitates 2738:1-50.

NATERA-MUMAW, M.; ESQUEDA, L.F.; CASTELAÍN, F.M. 2015. Atlas de serpientes de Venezuela. Una visión actual de su diversidad. Santiago de Chile, Chile.

PÉREZ-SANTOS, C.; MORENO, A.G. 1988. Ofídios de Colombia. Museo Regionale di Scienze Naturali, Torino 6:1-517.

PINZÓN, C.A. 2014. Análisis descriptivo y gráfico de datos climáticos. Restauración ecológica del Bosque Seco. Proyecto hidroeléctrico El Quimbo. Informe. Fundación Natura Colombia. .

PYRON, R.A.; BURBRINK, F.T.; WIENS, J.J. 2013. A phylogeny and revised classification of Squamata, including 4161 species of lizards and snakes. BioMed Central Evolut Biol. 13:93.

RANGEL-CH, J.O. 2004a. (ed.). Colombia Diversidad Biótica IV. El Chocó biogeográfico/Costa Pacífica. Instituto de Ciencias Naturales - Conservación Internacional. Bogotá D.C.

SAVAGE, J.M. 2002. The amphibians and reptiles of Costa Rica: A herpetofauna between two continents, between two seas. University of Chicago Press. Chicago, USA.

SUÁREZ, A.M.; ALZATE-BASTO, E. 2014. Guía llustrada Anfibios y reptiles Cañón del río Porce, Antioquia. EPM E.S.P. Universidad de Antioquia, Herbario Universidad de Antioquia. Medellín, Colombia.

TORRES-CARVAJAL, O.; YÁNEZ-MUÑOZ, M.H.; QUIROLA, D.; SMITH, E.N.; ALMENDÁRIZ, A. 2012. A new species of blunt-headed vine snake (Colubridae, Imantodes) from the Choco region of Ecuador. ZooKeys 244:91-110.

UETZ, P.; HOŠEK J. 2017. The Reptile Database. Disponible en: http://www.reptile-database.org. Consultado: 0504-2017.

VARGAS, Á.; GARCÍA-RODRÍGUEZ, J.; BATISTA, A.; ACOSTA-CHAVES, A.V.; BOLÍVAR, W.; CISNEROS-HEREDIA, D.F. 2016. Imantodes inornatus. The IUCN Red List of Threatened Species 2016: e.T176820A1448570. Disponible en: http://dx.doi.org/10.2305/IUCN.UK.2016-1.RLTS.T176820A1448570.en. Consultado: 19-12-2017.

ZHENG, Y.; WIENS, J.J. 2016. Combining phylogenomic and supermatrix approaches, and a time-calibrated phylogeny for squamate reptiles (lizards and snakes) based on 52 genes and 4162 species 94 (Parte B): $537-547$. 